\title{
ON CAPACITY MODELING FOR PRODUCTION PLANNING WITH ALTERNATIVE MACHINE TYPES
}

\author{
ROBERT C. LEACHMAN, TALI F. CARMON
}

Analyzing the capacity of production facilities in which manufacturing operations may be performed by alternative machin types presents a seemingly complicated task. In typical enterprise-level production planning models, capacity limitations of alternative machine types are approximated in terms of some single artificial capacitated resource. In this paper we propose procedures for generating compact models that accurately characterize capacity limitations of alternative machine types. Assuming that processing times among alternative machine types are identical or proportional across operations they can perform, capaciny limitations of the alternative machine types can be precisely expressed using a formulation that is typically not much larger than the basic lineas programming formulation that does not admit alternative resource types. These results have important in. plications for industrial practice, suggesting that in the case that processing times are nearly proportional among alternatives, the prevalent approximation that involves using a single, capacitated, artificial resource may be dropped in favor of our formulation incorporating the approximation that processing times among the alternatives are proportional. Another advantage is that the set of capacity constraints we formulate can be used to check the feasibility of suggested production schedules or demands simply by plugging them into the constraints, without need to develop values for allocation variables.

In this paper we present an efficient approach for formulating models of the capacity limitations of alternative machine types for use in corporate-level capacity analysis. In accordance with the hierarchical approach to production planning and control proposed by Gershwin [7], the corporate-level production planner is primarily interested in determining the optimal, capacity-feasible product mix for each planning period over some planning horizon, i.e., the optimal rates of production of each product type in periods such as weeks or months. One or more lower level planners (e.g., factory-floor schedulers) then determines the best way to produce this mix in a much shorter time frame such as a day, taking into consideration the detailed current status of the factory.

Lincar Programming (LP) is often proposed as a tool for production planning and scheduling [3], [4], [9], [11], [13]. However, LP formulations can be very large for large organizations with complex production environments, and care must be exercised to develop the most compact model possible achieving a desired level of accuracy. The microelectronics manufacturing industry is an example of such a complex manufacturing environment [1], [13], [15]. A large semiconductor firm must plan the production of thousands of products subject to capacity limitations imposed by hundreds of equipment types. In addition to the complexity caused by the varicty of processing requirements of the different product types, frequently there are alternative machine types suitable for performing manufacturing operations. (Throughout this paper we use the phrase "alternative machine types for an operation" to refer to machine types that are functionally different but nerertheless all suitable for performing the manufacturing operation. Such machine types have partial overlap in terms of the operations they can perform. We vie multiple identical machines as a single machine typ with given capacity.) Due to this equipment flexibility. higher throughput and capacity utilization can te achieved through balancing the workloads among the alternative machine types. However, analyzing the avait able capacity of this flexible environment in order to determine the optimal production quantities presents $\$$ seemingly complicated task.

If the high-level capacity analysis were to precisely characterize available capacity, one could imagine developing a detailed LP formulation in which the variables are defined as the amount of each product $n \mathrm{pt}^{t}$ taking each route through the factory (i.e., the work load assigned to a set of machines that perform a com plete sequence of operations on the product type). Thx total number of variables in each planning period would then be the number of all possible routes, for all prod. uct types. For example, if a product requires a single processing operation that has 4 alternative machine types, then 4 variables are required to represent it. Ho ever, if the route for the product incorporates this proessing operation twice, then 16 variables are requirol to represent all alternatives. Thus, in a re-entrant pro ess flow the number of variables grows as the numbt of alternative machine types for each operation to the power of the number of re-entries to that operation. 
The semiconductor industry is characterized by reentrant routes [1], [13], [15]. In the Lithography stage, for example, there are on the order of 10-20 re-entries, with several alternative machine types suitable for performing most lithography operations, but perhaps only one machine type suitable for critical operations. This is a typical case in which the number of variables in an LP formulation of this type would grow unattractively large.

In general, it is computationally cumbersome to carry out detailed assignments of operations to machines when the purpose at hand is to adjust company-wide demands to achieve capacity-feasibility. In typical enterprise-level planning applications, capacity limitations of alternative machine types are approximated using some sort of average resource capacity to constrain volumes of each product type to be produced, with varying degrees of success. For example, Spence and Welter [14] estimate a semiconductor manufacturing work cell's capacity using a cycle time-throughput trade off curve.

The problem of accurately modeling the capacity of ajternative, non-identical resources arises in almost all manufacturing environments where process technology is evolving, as well as in numerous other application areas. Surprisingly, published research on this topic is scant.

Semiconductor shop floor scheduling on alternative machine types has been addressed by Bitran and Tirupati [2]. Their goals were to minimize the schedule makespan and total tardiness. However, they considered a single stage process, and assigned jobs to machine types based on heuristic rules. Their approach is not suitable for high level planning of a multi-stage process. Federgruen and Groenevelt [5] find capacityfeasible schedules for $\mathbf{n}$ jobs with given release times and due dates. They use network flow techniques similar to ones we rely on, in that maximal throughput of jobs corresponds to a maximal flow in the network. However, they allow for preemption, and assume that al jobs can be processed on any of the machine types, albeit at different speeds.

In the next sections we develop alternative formulation approaches that are much more compact than the route-variable approach briefly discussed above. First, we develop a formulation that replaces variables representing entire production routes with variables representing production activity at each operation in a product's multi-stage production process. For each operation on each product, there are allocation variables to spread the product-operation production quantities among alternative machine types, and between consecutive operations there are constraints guaranteeing con- sistency of production volumes. This formulation, a natural extension of an approach suggested in [11], is termed the "Step-Separated Formulation" and is presented in the next section. This formulation is quite general but has the disadvantage of relatively large dimensions due to the explicit scheduling of machine assignments to product-operation volumes in order to determine capacity-feasibility.

We then introduce more compact models applicable under a uniformity assumption concerning the machines types. This assumption requires the processing times among alternative machines types to be either identical or else proportional across all operations performed by the machines. Exploiting this unifornity assumption. one of these formulations utilizes allocation variables for the total workload in planning periods rather than for the workloads of individual process- steps. We refer to such a formulation as the "Workload Allocation Formulation." We next show how an exact formulation may be generated without use of any allocation variables, i.e., including only variables for the production of each product. We term such a formulation the " $\mathrm{Di}$ rect Product Mix Formulation."

We also compare the different formulation techniques for a couple of examples that illustrate the range of dimensions that may result. We discuss the technological reasons underlying the existence of alternative machine types as they arise in industrial practice. We illustrate that for the most commonly arising sets of alternative machine types, the Direct Product Mix formulation is the most compact formulation by a substantial margin, and is typically not much larger than the conventional LP planning formulation that does not admit alternative resources. The restrictiveness of the uniformity assumption also is discussed. We explain the underlying technological factors that make this assumption a good approximation in semiconductor manufacturing. suggesting that the compactness of the proposed formulation is worth the loss of generality.

In all formulations we assume that the set of machine types suitable for performing a particular processing step is independent of the machine types selected to perform other steps on the same product. This assumption is typically realistic for semiconductor manufacturing. as well as for many other manufacturing environments.

We also assume that the production cost is independent of the product's processing route. This assumption is reasonable for semiconductor manufacturing since the incremental costs of raw material and labor are relatively small, and are typically considered identical or almost identical for all alternative machine types. Revenue is much larger than variable production cost, and 
therefore the major issue the production planner is concerned with is the utilization of production capacity.

\section{The Step-Separated Formulation}

We introduce the following notation for the data concerning the production planning problem:

$t=1, \ldots, \mathrm{T}$ is the time period index, where period $t$ denotes the time interval $(t-1, t]$,

$i=1, \ldots, n$ is the product type index,

$j=1, \ldots, J_{i}$ is the index of process-steps (operations) for product type $i$,

$L_{i j}$ denotes the average flow time for product type $i$ from the start of its production process until initiation of step $j$ (the "lead time" up to step $j$ ),

$L_{i}$ denotes the average flow time for product type $i$ from start to finish of its entire production process (the "lead time" for the process),

$k=1, \ldots, K$ is the machine type index,

$k \in P(i, j)$ denotes that machine type $k$ is suitable for performing step $j$ on product type $i$,

$a_{i j k}$ is the time required to process one unit of product type $i$ in step $j$ on machine type $k$,

$C_{k t}$ is the available capacity (in time units) of machine type $k$ in time period $t$,

$D_{i t}$ is the maximum cumulative number of units of product type $i$ that can be sold by time $t$ (i.e., the total market forecast, including both committed and potential future orders),

$d_{i r}$ is the minimum cumulative number of units of product type $i$ that must be supplied by time $t$ (i.e., the committed orders),

$p_{i r}$ is the estimated net discounted cash flow from producing and selling one unit of product type $i$ in time period $t$, and

$h_{i \text { is }}$ is the estimated cost of holding one unit of inventory of product type $i$ at time $t$.

For simplicity of exposition, we shall omit the effect on the formulations of various factors such as yields, initial status of work in process, and infeasibilities of given minimum demands. Extensions to account for such phenomena are addressed in detail in [13]. Also for sim'icity, we assume that all lead times are integer; in the case they are fractional, a formulation may be de. veloped in which step workloads and process outputs are split between adjacent periods. The lead times also can be time-varying, represented as parameters specific to epochs marking the end points of the planning $p$ riods. See [13] for details. All these extensions preserve the special structure developed here for coping with a. ternative resources.

All formulations we shall present include the follow. ing basic production and inventory variables:

$X_{i t}$ is the number of units of product type $i$ to be started in time period $t$,

$I_{i t}$ is the number of units of product type $i$ held in inventory at time $t$, and

$B_{i z}$ is the shortfall of the cumulative production vs. the cumulative max demand for product type $i$ at time?.

In the Step-Separated formulation, additional variables are introduced to represent the portion of the workload of each product type in each processing step in each time period that is assigned to each suitable ma. chine type. Formally, we let $W_{i j k t}$ denote the worklout of product type $i$ in step $j$ in time period $t$ that is assigned to machine type $k$, defined only for machine types $k \in P(i, j)$. The formulation may be expressed as follows:

$$
\operatorname{Max} \sum_{i=1}^{n} \sum_{t=1}^{T} p_{i t} X_{i r-L_{t}}-h_{i s} I_{i t}
$$

subject to

$$
\begin{gathered}
X_{i t-\Sigma_{i j}}=\sum_{k \in P(i, j)} \frac{W_{i j k t}}{a_{i j k}} \forall i=1, \ldots, n ; \\
\forall j=1, \ldots, J_{i} ; \forall t=1, \ldots, T
\end{gathered}
$$

(for each $i$, one of these expressions could be substituted to replace $X_{i t-z_{i j}}$ )

$$
\sum_{\substack{i=1 \\\{i, j \mid k \in \mathcal{j} \in(i, j)\}}}^{n} \sum_{\substack{j \\ i}}^{s_{i j k t}} \leq C_{k t} \forall k=1, \ldots, K ; \forall t=1, \ldots, T
$$

$\sum_{\tau=1}^{t} X_{i \tau-L_{i}}-I_{i \mathrm{t}}+B_{i \mathrm{t}}=D_{i \mathrm{t}} \forall i=1, \ldots, n ; \forall t=1, \ldots, T^{-1}$

$$
\sum_{r=1}^{T} X_{i \mathrm{~T}-L_{i}}+B_{i \mathrm{~T}}=D_{i T} \forall i=1, \ldots, n
$$

$$
B_{i t} \leq D_{i t}-d_{i t} \forall i=1, \ldots, n ; \forall t=1, \ldots, T
$$

$X_{i s} \geq 0, I_{i \mathrm{r}} \geq 0, B_{i t} \geq 0, W_{i j k t} \geq 0$, for all $i, j, k_{t} !$

Note that the formulation assumes no intermediate accumulation of inventory is allowed, i.e., only finisher goods may be stored. The formulation allows outpot 
illing the portion of market forecasts in excess of nmitted orders $\left(D_{i t}-d_{i t}\right)$ to be delivered late, albeit less discounted revenue. In the objective function this and all subsequent formulations, discounted net h flow is credited against production, even though s production might not be immediately sold. Howir, we assume the inventory holding costs include : difference in discounted sales revenue from one pe$x d$ to the next. Since ending inventory is prohibited, rect discounted net cash flow is assessed overall. te that in the Step-Separated formulation, an addimal set of constraints is required to guarantee that r each product type, the number of units processed each step is consistent with the start quantity. The uacity constraints then force the total workload on th machine to be less than or equal to the machine ipacity.

\section{iumber of Variables and Constraints}

Let $M_{i j}$ be the number of alternative machine types at can perform step $j$ on product type $i$. Excluding iventory and backorder variables that will be common o all formulations we shall present, the number of varables per time period in the Step-Separated formulation

$$
\sum_{i=1}^{n} \sum_{j=1}^{J_{i}} M_{i j}
$$

htile the number of capacity-related constraints per time xeriod is

$$
K+\sum_{i=1}^{n}\left(J_{i}-1\right)=K-n+\sum_{i=1}^{n} J_{i}
$$

ince in addition to the $K$ machine capacity constraints, $1,-1$ equality constraints are required for each product pe $i$ to guarantee that the number of units processed it each step is consistent with the start quantities. These imensions are somewhat reduced in the degenerate case there some $M_{i j}$ 's are unity, i.e., in the case that there tre some operations with only a single suitable machine pe for which alocation variables are not needed. (We mit discussion of the dimensions of the demand conuraints since these constraints are identical for all fornulations to be presented.)

In the above formulation, the allocation variables repesent assignments of volumes to machines. The formulation is inefficient in the sense that it actually solves he detailed problem of allocating process operation orkloads among alternative machine types, yet the roduct start rates $\left(X_{i x}\right.$ 's) are the only variables truly thired at the highest level of the planning and scheding hierarchy. At this level of planning, it is not relistic to develop a detailed, operation-by-operation enterprise-wide schedule that one expects to be precisely followed; but it is nevertheless important to develop starts rates that are capacity-feasible based on average rates of available capacity. In typical practice, the operation-level scheduling problem would be solved much more frequently and with a shorter time horizon, whereby its formulation would reflect more refined knowledge about the state of the factory (c.g., machine availability and work in process) than can be reflected in the average rates assumed in high-level planning. That is, the corporate level production planner views shop floor activities as rates, and, under certain assumptions, as long as the shop floor data used by the high level planner is accurate, a feasible high level production plan can always be translated into a feasible schedule on the shop floor [7].

In the next section we show that it is possible to formulate the production planning problem in a more efficient manner. In lieu of variables representing allocations of the workload of individual process-steps, variables are defined to represent the allocation among machine types of the total workload that can be performed by a set of machine types. This formulation requires a uniformity assumption, specifically, that processing times among alternative machine types are either identical or else proportional across all operations performed by the machine types.

\section{The Workload Allocation Formulation}

If all alternative machine types have identical processing times, then the workload in a given time period for the set of process-steps that can utilize any of a set of alternative machine types may be summed to express the total workload in the time period on the set of machine types. If instead the processing times on alterna. tive machine types are proportional across all operations the alternatives perform, the processing times and capacities for the machine types may be scaled in terms of some "standard" machine type to achicve identical processing times and thereby obtain the same resuit. This assumption allows a substantial simplification of the Step-Scparated formulation. replacing allocation variables for individual process-steps with allocation variables for the total workload in a time period. We develop this formulation as follows. (We are indebted to Professor Ilan Adler for suggesting this formulation.)

When the uniformity assumption holds, the $k$ index of the $a_{i j k}$ time coefficients can be climinated since these coefficients no longer depend on the machine type selected for processing step $j$ of product type $i$. The following additional notation is required for this formula- 
tion:

$S_{m}, m=1, \ldots, M$, denote the unique sets of alternative machine types among the sets $P(i, j)$ appearing in the problem data,

$Z_{k t}^{m}$ is a variable representing the workload on set $S_{m}$ assigned to machine type $k$ in period $t$, defined for each $k \in S_{m}$.

The Workload Allocation formulation is then expressed as follows:

$$
\operatorname{Max} \sum_{i=1}^{n} \sum_{t=1}^{T} p_{i t} X_{i t-\bar{I}_{i}}-h_{i t} I_{i t}
$$

subject to

$$
\begin{gathered}
\sum_{\substack{i=1 \\
\text { i, } \\
\left\{i, j \mid P(i, j)=S_{m}\right\}}}^{J_{i}} a_{i j} X_{t t-L_{i j}}=\sum_{k \in S_{m}} Z_{k t}^{m} \quad \forall m ; \forall t \\
\sum_{m=1}^{M} Z_{k t}^{m} \leq C_{k t} \quad \forall k ; \forall t \\
\sum_{\tau=1}^{L} X_{i r-L_{i}}-I_{i t}+B_{i t}=D_{i t} \forall i=1, \ldots, n ; \forall t=1, \ldots, T-1 \\
\sum_{\tau=1}^{T} X_{i r-L_{i}}+B_{i T}=D_{i T} \quad \forall i=1, \ldots, n \\
B_{i t} \leq D_{i t}-d_{i t} \quad \forall i=1, \ldots, n ; \quad \forall t=1, \ldots, T
\end{gathered}
$$

$X_{i t} \geq 0, I_{i t} \geq 0, B_{i t} \geq 0, Z_{k t}^{m} \geq 0$, for all $i, j, k, m, t$.

\section{Number of Variables and Constraints}

The number of capacity constraints per time period in the Workload Allocation formulation depends on the number of sets of alternative machine types that appear in the problem data. Similarly, the number of variables per time period appearing in the capacity constraints depends on the cardinality of each of these sets. To express capacity limitations in each time period, the formulation includes $K$ inequality constraints, one for the capacity of each machine type, plus $M$ equality constraints relating the allocation variables for each machine set to the workload for the set. The number of equality constraints may total to slightly less than $M$ when there are one or more sets $S_{m}$ that are singletons. Examples may be constructed for which $M$ can range from $K-1$ to $2^{K}-K-1$, but for most industrial cases we have examined it is $O(K)$ or $O\left(K^{2}\right)$. As for the number of variables, let

$$
M_{t}=\sum_{\substack{m=1 \\\left|S_{m}\right| \geq 2}}^{M}\left|S_{m}\right|
$$

The Workload Allocation formulation includes $n+M$, variables appearing in capacity constraints in each tis period. We remark that $\left|S_{m}\right|$ is typically $O(K)$; hex $M_{t}$ is typically $O\left(K^{2}\right)$ or $O\left(K^{3}\right)$.

\section{The Direct Product Mix Formulation}

For the assumptions of the previous section, it is $p_{\text {s }}$ sible to construct an exact formulation of the capact limitations without using any allocation variables at al We call such a formulation the Direct Product Mixfue mulation. This formulation is developed by introductin capacity constraints for sets of alternative machine tyet The particular sets that need to be included depend a the machine usage patterns appearing in the proble data, whereby the total number of sets required $\mathrm{mit}$ turn out to be as small as the number of different tit chine types or as large as the power set of all machis? types. The justification for this formulation is based o an examination of the allocation problem in terms $d$ Duality Theory for Network Flows. Before plungin: into this theoretical justification, we summarize the COR struction of the Direct Product Mix formulation as foilows. First, a procedure we shall term the Capacity st Generation Procedure is used to identify the sets of $m$ chine types to be represented with corresponding ${ }^{c}$ pacity constraints in the formulation. Basically, th specification of such a capacity constraint involves th definition of two sets: a set of machine types whos capacities are summed to form the right hand side of the constraint, and a set of operations (i.e., product steps) whose loads are summed to form the left-han side of the constraint. The procedure develops thes sets as follows:

Capacity Set Generation Procedure

Step 1. Identify all sets of alternative machine types appearing in the problem data that are suitable for performing one or more product-steps. $\mathrm{C}$. pacity constraints will be formulated for each of these identified sets. The set of operations for this constraint will include all operations that load the identified set of machine types of ? any proper subset. The capacity of the set machine types for this constraint is the sum of the scaled capacities of the machine types in the identified set.

Step 2. For all sets of machine types identified in Sttp? 1 that have elements in common, form unions of these sets of machine types and unions of the operations that would appear in the corm. sponding constraints. Constraints are also for mulated for all new, larger sets so formed. 
Step 3. Continue this process of forming unions of intersecting sets of machine types so as to combine sets created in Step 2 with each other or with sets identified in Step 1, terminating when no new distinct sets of machine types can be generated.

Let $S$ denote an arbitrary set of machine types generated by this procedure. We write $(i, j) \in S$ to denote that the load from performing $j$ for product $i$ is included in the left hand side of the capacity constraint for set $S$, and we write $(k) \in S$ to denote that the capacity of machine type $k$ is included in the right hand side of the capacity constraint for set $S$. The resulting Direct Product Mix formulation can then be written as follows:

subject to

$$
\operatorname{Max} \sum_{i=1}^{n} \sum_{i=1}^{T} p_{i t} X_{i t-L_{i}}-h_{i t} I_{i t}
$$

$$
\begin{gathered}
\sum_{i=1}^{n} \sum_{(i, j) \in S} a_{i j} X_{i t-x_{i j}} \leq \sum_{(k) \in S} C_{k t} \text { for all generated sets } S ; \forall t \\
\sum_{r=1}^{t} X_{i r-L_{i}}-I_{i t}+B_{i t}=D_{i t} \forall i=1, \ldots, n ; \forall t=1, \ldots, T-1 \\
\sum_{i=1}^{T} X_{i \tau-L_{i}}+B_{i T}=D_{i T} \quad \forall i=1, \ldots, n \\
B_{i t} \leq D_{i t}-d_{i t} \quad \forall i=1, \ldots, n ; \quad \forall t=1, \ldots, T \\
X_{i t} \geq 0, I_{i t} \geq 0, B_{i t} \geq 0, \text { all } i, \text { all } t .
\end{gathered}
$$

\section{Number of Variables and Constraints}

Defined this way, the number of variables appearing in capacity constraints each time period is equal to $n$, the number of product types, which is always smaller than the number of variables in the previous approaches. The number of capacity constraints per period is the number of generated sets $S$ of machine types, which depends on the usage patterns appearing in the problem data. Two simple examples discussed in the next section illustrate that this number could be as small as $K$, or as large as $2^{x}-1$. As will be discussed in a later section, for most practical cases, this number is close to the number of machine types $K$, making this formulation very attractive. In fact, the formulation is usually only slightly larger (in terms of the numbers of variables and constraints) than the standard LP planning formulation that does not admit alternative resource types.

\section{Theoretical Justification}

The capacity feasibility conditions used in the Direct Product Mix formulation are justified using a modifi- cation and application of a feasibility theorem for Transshipment [12] Network Flows. We now transform the capacity limitations of the production planning problem into a Transshipment (more specifically, a Transportation) network representation, whereupon we shall state the feasibility theorem and formally develop its application to the construction of capacity constraints in the Direct Product Mix formulation.

Network Representation of the Capacity Limitations

A transportation-type network representing the capacity limitations of the production planning problem in an arbitrary time period $t$ in the case of alternative machine types is depicted in Figure 1 . Let $(i-j)$ be a node representing step $j$ of product type $i$, and let $(k)$ be a node representing machine type $k$. An arc $(k, i-j)$ from node $(k)$ to node $(i-j)$ indicates that step $j$ of product type $i$ can be processed on machine type $k$. These two sets of nodes and the arcs connecting them form a bipartite graph $G$ which may have one or more components (a component of $G$ is a connected subgraph of $G$ that is not connected to other subgraphs of $G$, i.e., the components of $G$ determine a unique partition of its nodes and arcs [12]). Let $A$ be the set of arcs in the network. The labels $\left(L_{a b}, U_{a b}\right)$ indicate the minimal and maximal flows allowed on arc $(a, b)$. The labels on the source nodes on the left denote available inflows, while the labels on the sink nodes on the right denote required outflows to be explained below. Note that the condition that the processing time coefficients $a_{i j k}$ are independent of $k$ is necessary for the network representation of Figure 1. Otherwise, multiple nodes are required to describe each step of each product type-a node for each of the alternative machine types.

The exogenous outflow $V_{i j}$ requirements in the network can be identified as the total workload in time period $t$ for step $j$ of product type $i$ (which in the formulations is expressed in terms of the total production of product type $i$, i.e., $\left.V_{u}=a_{i j} X_{i-t_{u}}\right)$. The $C_{k}$ inflows to the network represent the (known and limited)

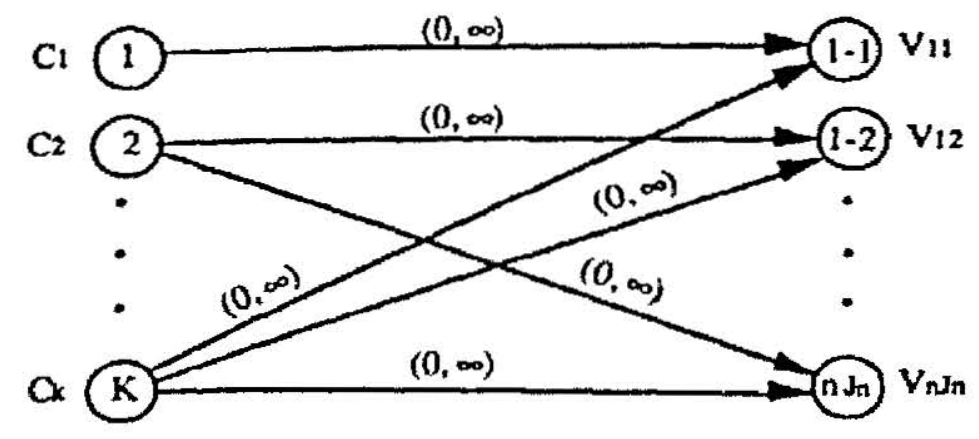

Figure 1: Network representation of the capacity limitations 
capacities of the machine types in period $t .\left(C_{k t}\right.$ 's in the formulation). The $W_{i j k t}$ variable of the Step-Separated formulation, which represents the workload of step $j$ for product type $i$ assigned to machine $k$, can be identified as the flow on the arc $(k, i-j)$, and the capacity constraints of the Step-Separated formulation, which guarantee that the total workload assigned to each machine does not exceed the machine capacity, correspond to the flow conservation constraints at the machine type nodes. (We omit from the network a dummy sink node for absorbing excess capacities.)

Note also that if the $X_{i t}$ 's were known, the $V_{i j}$ 's would be known, and the decision on assignment of workload to machines would become the Transportation [9] problem. Thus the capacity-feasibility of an arbitrary set of $X_{i t}$ 's may be evaluated in terms of the conditions for a feasible solution of the underlying Transportation problem.

\section{Applying Gale's Flow Feasibility Theorem}

There are several flow feasibility theorems that can be applied to the foregoing network. All of these theorems are specialized versions of the general max-flow min-cut theorem. Hoffman's Circulation Theorem [10], [12] expresses feasibility conditions for general networks. Hall's Theorem [8] is a special case that applies to bipartite graphs for the $0-1$ assignment problem, and perhaps could be extended to apply to the problem at hand. However, it is most convenient to make use of Gale's Flow Feasibility Theorem for Transshipment Networks [6], of which Transportation Networks are a special case.

Gale's Feasibility Theorem states (in our notation) that a Transshipment problem is feasible if and only if

$$
\sum_{(i-j) \in S} V_{U} \leq \sum_{(k) \in S} C_{k}+\sum_{(k, i-j) \in(T, S)} U_{k, i-j}
$$

for all cutsets $(S, T)$. Here, a cutset is defined as a partition of the set of all nodes into two nonempty, mutually exclusive subsets $S$ and $T$. We now apply this theorem to generate capacity constraints on the $X_{i t}$ variables. We shall start by identifying what we shall term the dominant cutsets of the network which are cutsets that correspond to the required capacity constraints. We then demonstrate the redundancy of all other cutsets. Formally, we define the dominant $(S, T)$ cutsets as those cutsets of the set of product-step and machine nodes that satisfy the following conditions:

1. If $(i-j) \in S$ then $(k) \in S \quad \forall(k, i-j) \in A$

2. If $\exists\left(i-j^{*}\right)$ such that whenever $\left(k, i-j^{*}\right) \in A$ we have $(k) \in S$, then $\left(i-j^{*}\right) \in S$
3. $S$ is connected.

Proposition (Elimination of Dominated Cutset:

Cutsets for which one or more of the conditions a are violated are dominated in the sense that the $c$ tions of the flow feasibility theorem are automati satisfied.

\section{Proof:}

1. If $(i-j) \in S$ and $\exists(k) \in T$ s.t. $(k, i-j) \in A$ then $s$ $U_{k, i-j}=\infty$ we get

$$
\sum_{i-j \in S} V_{i j} \leq \infty
$$

which is always satisfied.

2. If $\exists\left(i-j^{*}\right) \in S$ such that $(k) \in S \forall(k)$ s.t. $\left.\left(k, i-j^{*}\right)\right\}$ then the resulting constraint is

$$
\sum_{(i-j) \in S} V_{u} \leq \sum_{(k) \in S} C_{k}
$$

which is dominated by the constraint resulting fi adding $\left(i-j^{*}\right)$ to $S$, i.e.,

$$
\sum_{(i-j) \in S} V_{i j}+V_{i j *} \leq \sum_{(k) \in S} C_{k} .
$$

3. If $S$ is not connected then without loss of genera let $S^{\prime}$ and $S^{\prime \prime}$ represent two subsets of $S$ s.t. $S^{\prime}+$ $=S, S^{\prime}$ is connected and $S^{\prime \prime}$ is connected. The sulting constraint is

$$
\sum_{(i-j) \in S^{\prime}} V_{i j}+\sum_{(i-j) \in S^{*}} V_{i j} \leq \sum_{(k) \in S^{\prime}} \mathcal{C}_{k}+\sum_{(k) \in S^{*}} \mathcal{C}_{k}
$$

which is dominated by the constraints

$$
\begin{aligned}
\sum_{(i-j) \in S^{\prime}} V_{i j} & \leq \sum_{(k) \in S^{*}} C_{k} \\
\sum_{(i-j) \in S^{*}} V_{i j} & \leq \sum_{(k) \in S^{*}} C_{k}
\end{aligned}
$$

Applying the flow feasibility theorem to the dom cutsets, we obtain a set of inequalities of the for

$$
\sum_{(i-j) \in S} V_{i j} \leq \sum_{(k) \in S} C_{k}
$$

i.e., in the appropriate form to serve as LP caf constraints, where the $V_{i j}$ 's are the workloads in by the LP variables (the $X_{i t}$ variables defined representing the feasible amounts of product type can be processed, and the $C_{k}$ 's are the machin capacities (the $C_{k r}$ 's in the formulation). Interp the definition of a dominant cutset, note that it $d$ a set of machine types and a set of product-step actly those product-steps that can be performed or more of the defined machine types are include 
connectedness of $S$ indicates that $S$ is defined in terms of a set of machine types that with respect to one another are all alternative types for some particular product-step, or else $S$ is defined as a union of such sets that have one or more elements in common. It should now be clear that the Capacity Set Generation Procedure introduced at the beginning of this section is precisely the procedure for generating the sets of machine types and product-steps that correspond to the dominant cutsets.

Assuming the input data is sorted by product-steps and by machine types, the theoretical complexity of the Capacity Set Generation Procedure is $O\left(P N+P 2^{K}\right)$, 2here $P$ is the maximal cardinality of the alternative machine sets, $K$ is the number of the machine nodes of the maximal (in terms of number of the machine modes) connected component of the bipartite graph of product-steps and machine types, and $N$ is the number of product-steps in the problem data. The first step of the procedure requires an $O(P N)$ sorting operation to eliminate multiple identical machine sets and identify of the procets and their subsets. The second and third steps is $O\left(P 2^{K}\right.$. is $O\left(P 2^{K}\right)$. Note that although the Procedure is exponential, it is practically very useful since $K$, the maxof the bipart of machine types in a connected component of the bipartite graph, is typically a relatively small number, as explained in the next section.

\section{Comparison of the Formulations}

At the outset it should be noted that the constraints of the Direct Product Mix formulation have much wider application than just inclusion in linear programming models. The capacity-feasibility of any proposed set of start rates $\left(X_{i i}\right.$ 's) may be checked by simply substituting the proposed schedule into these constraints. In contrast, the Step-Separated and Workload Allocation formulations require one to develop optimal values for allocation variables to assess the capacity-feasibility of proposed start rates.

It is also important to note that in most industrial enterprises, the overall data set describing capacity limitations can be broken down into separate data sets describing several independent groups of machine types. For example, a semiconductor wafer fabrication facility might have capacity data concerning photolithography machines, ion implant machines, plasma etch machines, etc., whereby each group performs different kincts of processing steps. The capacity constraints for cach group may be constructed separately, and then combined to form the complete set of constraints. For each independent group, $K$ will be typically a relatively small number, e.g., 1-4. Thus, we are primarily interested in studying the size of formulations with small values of $K$. The number of products and the number of process-steps per product is perhaps more variable from enterprise to enterprise. For semiconductor wafer fabrication facilities we have studied, the number of product types $n$ ranges from $10-300$, while the average number of process steps per product per independent group of machine types ranges from as low as one for certain kinds of deposition equipment to a high of 18 for the photolithography group. An enterprise-level planning model may involve dozens of manufacturing facilities, thousands of products and hundreds of machine types, but nevertheless the overall formulation of capacity limitations may be developed by combining formulations for relatively small, independent groups of machine types.

We now compare the dimensions of the StepSeparated, Workload Allocation and Direct Product Mix formulations. As noted before, the precise dimensions depend on the particular machine usage pattern cvident in the problem data. We shall focus on a couple of simple examples that concisely illustrate the range of dimensions of these formulations that may be encountered. For each example, we shall note the number of constraints, variables and nonzero elements in each type of formulation. We make the assumption that all products have one or more process-steps that load each set of alternative machine types appearing in the data. For simplicity of exposition of the results for the StepSeparated formulation, we further assume that for each product there are exactly $J$ process-steps (operations) loading one or more of the $K$ machine types, and that the assignment of process-steps to sets of machines is uniformly distributed.

In the first example, suppose the only sets of alternative machine types appearing in the problem data are the singleton machine type $A$, the pair of machine types $A$ and $B$, the set of three machine types, $A, B$, and $C$, and so on, up through the set of all $K$ machine types. That is, the sets of alternative machine types for the various process-steps can be arranged as a series of nested subsets. As will be discussed in the next section. this is the most common pattern of alternatjve machine sets in semiconductor manufacturing. The middle column of Table 1 displays dimensions of the formulations of this example. As can be seen, the Step-Separated formulation pays a heavy price for the generality of non. uniform processing times, with the number of capacityrelated constraints per time period $O(n J+K)$. and the number of variables appearing in these constraints 


\begin{tabular}{|c|c|c|}
\hline \multirow[t]{2}{*}{ FORMULATION TYPE } & \multicolumn{2}{|c|}{ PKOBLEM TYPE } \\
\hline & Nested Sets & All Singletons and All Pairs \\
\hline \multicolumn{3}{|l|}{ Step Separated } \\
\hline Constraints & $\left(1-\frac{1}{K}\right) n J+K=O(n J+K)$ & $n J \frac{\left(\begin{array}{l}K \\
2\end{array}\right)}{K+\left(\begin{array}{l}K \\
2\end{array}\right)}+K=O(n J+K)$ \\
\hline Variables & $\frac{n J}{K}\left\{\frac{K(K+1)}{2}-1\right\}+n=O(n J K)$ & $2 n J \frac{\left(\begin{array}{l}K \\
2\end{array}\right)}{K+\left(\begin{array}{l}K \\
2\end{array}\right)}+n=O(n J)$ \\
\hline Nonzeros & $n J\left(K-\frac{3}{K}+2\right)+n=O(n J K)$ & $n J(n+4) \frac{\left(\begin{array}{l}K \\
2\end{array}\right)}{K+\left(\begin{array}{l}K \\
2\end{array}\right)}+n K=O\left(n^{2} J+n K\right)$ \\
\hline \multicolumn{3}{|l|}{ Workload Allocation } \\
\hline Constraints & $2 K-1$ & $K+\left(\begin{array}{l}K \\
2\end{array}\right)=O\left(K^{2}\right)$ \\
\hline Variables & $n+\left\{\frac{K(K+1)}{2}-1\right\}=O\left(n+K^{2}\right)$ & $n+2\left(\begin{array}{l}K \\
2\end{array}\right)=O\left(n+K^{2}\right)$ \\
\hline Nonzeros & $n K+2\left\{\frac{K(K+1)}{2}-1\right\}=O\left(n K+K^{2}\right)$ & $n\left[K+\left(\begin{array}{l}K \\
2\end{array}\right)\right]+4\left(\begin{array}{l}K \\
2\end{array}\right)=O\left(n K^{2}\right)$ \\
\hline \multicolumn{3}{|l|}{ Direct Product Mix } \\
\hline Constraints & $K$ & $2^{K} \cdot 1$ \\
\hline Variables & $n$ & $n$ \\
\hline Nonzeros & $n K$ & $n\left(2^{K}-1\right)$ \\
\hline
\end{tabular}

Table 1. Comparison of Formulation Dimensions for the Case of Nested Sets and the Case of All Singletons and All Pairs (Number of Capacity-Related Constraints per Timer in these Constraints)

$O(n J K)$. Capacity-related constraints dominate demand constraints in this formulation by a factor of $J$. For large values of $n$ and $J$, this formulation is prohibitively large.

In contrast, the Direct Product Mix formulation in each time period has the bare minimum number of capacity constraints (the number of machine types $K$ ) and the bare minimum number of variables (the number of product types $n$ ). It is essentially no different in its matrix dimensions than the basic LP formulation that does not admit alternative machine types. (This result is obtained because Steps 2 and 3 of the Capacity Set Generation Procedure do not generate any new distinct sets for the nested-subset structure.)

Compared to the Direct Product Mix formulation, the Workload Allocation formulation for this case includes almost double the number of capacity-relater constraints per time period (specifically, $2 K-1$ ), with an additional $O\left(K^{2}\right)$ variables appearing in these constraints.
The number of nonzero elements also is larger by at additive factor of $O\left(K^{2}\right)$.

The last column of Table 1 displays results for at example that yields the worst-case machine usage pat tern for the Direct Product Mix formulation, i.e., a ts age pattern that leads to the power set when the Capaciti Set Generation Procedure is applied. To obtain this sult requires a somewhat pathological example in which every machine type and every combination of two ms. chine types each appear as a suitable set of machint types for at least one operation (the case of "All Sint gletons and All Pairs"'). In such a case, Steps 2 ax 3 of the Set Generation Procedure will generate all un ons of $3,4, \ldots, K$ machine types, and the resulting num ber of capacity constraints per time period is

$$
\sum_{i=1}^{k}\left(\begin{array}{c}
K \\
i
\end{array}\right)=2^{\kappa}-1
$$


Employing the Workload Allocation formulation to develop capacity constraints for this example, this formulation generates $O\left(K^{2}\right)$ capacity constraints per time period on $O\left(n+K^{2}\right)$ variables. For small $K$ in this problem type, the two formulations are comparable, but the Workload Allocation formulation is preferred when $\boldsymbol{K}$ is 4 or more. With respect to translating these comparisoas into optimization run-time comparisons, it should be remembered that the capacity constraints in these two formulations typically account for a much smaller portion of the overall constraint matrix than do the demand constraints, which are identical among the alternative formulations.

The Step-Separated formulation for this example still has the very unattractive features whereby the number of variables and the number of capacity-related constraints are proportional to $n J$.

\section{Alternative Machine Types Arising in Semiconductor Manufacturing}

In a company-wide capacity model for semiconduclor manufacturing that the first author developed, the resulting number of capacity constraints per time period when the Direct Product Mix formulation was applied turned out only slightly larger than the total number of resources $K$. To understand this result requires an explanation of the underlying technological factors giving rise to alternative machine types in this industry. The theme underlying the vast majority of cases of alternative machine types is technological progress, whereby the alternative machine types can be ordered in terms of capability. The most common attribute of technological progress is machine precision. For example, the newest machine type may be capable of precision to very fine geometries, making it eligible to perform operations with the most critical geometries, as well as all other operations. Older machine types may be unable to perform the most critical operations. After several generations of progressively improved machine types have been installed, the factory finds itself in a state such that only the highest precision machine type can be used for the most critical operations, the best two types can be used for the next most critical set of operations, and so forth, down to the set of least critical operations which may be performed on any of the alternative machine types. As we have seen, the number of capacity constraints per time period for the Direct Product Mix formulation in these cases is exactly $K$, with only the n planning variables appearing in them. For nested set patterns of machine usage, it is clear that the Direct Product Mix formulation is preferred from the point of view of formulation size. In applying the Direct Product Mix formulation to more than 100 independent groups of alternative machine types, pathological examples leading to the complete power set explosion of constraints, such as the all-singletons-andall-pairs-of-types example discussed in the previous section, were never encountered for values of $K$ larger than 2.

The remarkable economy of the Direct Product Mix formulation relies on the assumption of uniformity of processing times among alternative machine types, so it is of interest to know how reasonable this assumption is in industrial practice. Reviewing processing time data from many semiconductor factories, we find that the proportionalities of processing times for alternative machine types are typically very similar but not exactly constant across different processing steps. Again, there are technological reasons underlying this empirical result. The overall processing time of an operation consists of two parts: a true machine processing portion, and a generally much smaller portion devoted to material handling just before and just after true processing activity. A new generation of equipment occasionally will proportionally improve the speed of true processing, but continue to use the same material handling technology. Since the handling activity is only a small portion of total processing time, the overall processing times for old and new machine types are approximately proportional.

A good approximation of this situation may be developed by computing weighted-average ratios of the processing times among alternative machine types, and then converting the processing time and capacity data into equivalents for a machine type arbitrarily selected as the "standard." Thus the Direct Product Mix formulation of the planning problem may be employed with only relatively minor loss of accuracy.

\section{Conclusions and Further Research}

In this paper we have analyzed techniques for formulating capacity constraints describing the limitations of alternative machine types in corporate-level production planning models. We have proposed the Direct Product Mix formulation for this purpose, justified using a network representation of the process and a flow feasibility condition that determines the required set of capacity constraints. The main advantage of this approach is that under a uniformity assumption concerning processing times, capacity limitations of alternative machine types can be precisely expressed using a formulation that is typically comparable in size to the basic 
LP formulation that does not admit alternative resource types. These results have important implications for industrial practice, suggesting that in the case that processing times are nearly proportional, the prevalent, crude approximation that represents capacity of alternative machine types in terms of an artificial, average resource may be replaced by the refined approximation that processing times among the alternatives are proportional. Another advantage is that the resulting set of capacity constraints can be used to check the feasibility of suggested production schedules or demands simply by plugging them into the constraints, without need to develop values for allocation variables.

For large numbers of alternative machine types that do not appear as nested sets in the problem data, the Workload Allocation formulation may be preferred. Cases where this formulation is preferred seem to be rare in practice.

The problem of formulating the capacity constraints for alternative resources that are not independent (e.g., alternative tools and alternative machines used at the same time) is not treated in this article. This problem is very common in semiconductor testing operations and is the subject of current research.

\section{Acknowledgements}

This research was supported by grants to the University of California at Berkeley from Harris Corporation-Semiconductor Sector and from Semiconductor Research Corporation. We are grateful to Harris Corporation-Semiconductor Sector for the opportunity to study capacity data from more than $\mathbf{2 0}$ semiconductor manufacturing facilities.

\section{REFERENCES}

[1] Bai, X., and Gershwin, S. B., “A Manufacturing Scheduler's Perspective on Semiconductor Fabrication,' 'Laboratory for Manufacturing and Productivity, MIT (1989).

[2] Bitran, G. R., and Tirupati, D., "Planning and Scheduling for Epitaxial Wafer Production Facilities," Operations Research, Vol. 36, No. 1, pp 34-49 (1988).

[3] Burman, D. Y., Gurrola-Gal, F. J., Nozari, A., Sathaye, S., and Sitarik, J. P., "Performance Analysis Techniques for IC Manufacturing Lines," AT\&T Technical Journal, Vol. 65, No. 4, pp 46-56 (1986).

[4] Dantzig, G. B., Linear Programming and Extensions, Princeton University Press, Princeton, NJ (1967).
[5] Federgruen, A., and Groenevelt, H., "Preemptive Schoduty of Uniform Machines by Ordinary Network Flow Techripks. Management Science, Vol. 32, No. 3, pp 341-349 (1936).

[6] Gale, D., The Theory of Linear Economic Models, MiCrs. Hill Publishing Co., New York, NY (1960).

[7] Gershwin, S. B., "Hierarchical Flow Control: a Framendi for Scheduling and Planning Discrete Events in Manufacuris Systems," IEEE Proceedings of Special Issue on Discrete fitu Systems (1988).

[8] Hall, P., "On Representatives of Subsets," J. London W.9. Society, Vol. 10, pp 26-30, 1935.

[9] Hillier, F. S., and Lieberman G. J., Introduction to Operwix Research, McGraw-Hill Publishing Co., New York, NY(IMx)

[10] Hoffman, A. J., "Some Recent Applications of the Thang Linear Inequalities to Extremal Combinatorial Analysis," hn ceedings of Symposium on Applied Mathematics, Vol. 10 (1969.

[11] Johnson, L. A., and Montgomery, D. C., Operations Resin? in Production Planning, Scheduling, and Inventory Control, bot Wiley \& Sons, New York, NY (1973).

[12] Lawler, E. L., Combinatorial Optimization: Netwon'sads troids, Holt, Rinehart, and Winston, New York, NY $19 \%$

[13] Leachman, R. C., "Modeling Techniques for Global Prota tion Planning in the Semiconductor Industry," ESRC Rn? 92-7, Engineering Systems Research Center, University of 6 , ifornia, Berkeley (1992), to appear as a chapter in Optinizen in Industrial Environments, John Wiley \& Sons, New int NY (1992).

[14] Spence, A. M., and Welter, D. J., "Capacity Plannimes $(1$. Photolithography Work Cell in a Wafer Manufacturing lix. Proceedings of the IEEE International Conference on $R$ ht and Automation (1987).

[15] Uzsoy, R., Lee, C. Y., and Martin-Vega, L., "A Revitr t Production Planning and Scheduling Models in the Semicnis tor Industry," Research Memorandum No. 90-11, Purdut ti versity (1991). 